\title{
Evaluación de dos Piretroides en el Control del Vector del Dengue en Putumayo, Colombia
}

\section{Evaluating two pyrethroids in dengue vector control in Putumayo, Colombia}

\author{
Mirley Castro ${ }^{1}$, Nilson Quintana² y Martha L. Quiñones $\mathrm{P}^{3}$.
}

1. Facultad de Medicina, Universidad de Antioquia. Medellín, Colombia. castrosalas@gmail.com

2. Dirección Territorial de Salud de Caldas. Manizales, Colombia. nilsonquintana@gmail.com

3. Departamento de Salud Pública, Facultad de Medicina, Universidad Nacional de Colombia,

Bogotá. mlquinonesp@unal.edu.co

Recibido 30 Junio 2006/Enviado para Modificación 26 Noviembre 2006/Aceptado 3 Enero 2007

\section{RESUMEN}

Objetivo Evaluar la eficacia de dos insecticidas piretroides sobre Aedes aegypti, información útil en la toma de decisiones para el control de dengue.

Métodos Se evaluaron los piretroides highcis-Permetrina (Depe $®)$ en aplicación en ultra bajo volumen (ULV) y ß-cipermetrina en un pote fumígeno (Bolate ${ }^{\circledR}$ ) sobre A. aegypti en 16 manzanas de la cabecera municipal de Puerto Leguízamo, Putumayo, entre Julio y Diciembre del 2004. El efecto inmediato se midió en jaulas centinela colocadas en diferentes sitios de las viviendas y se realizó un seguimiento semanal evaluando las densidades de adultos y presencia de larvas.

Resultados La reducción de las densidades de adultos el día posterior a la aplicación de los insecticidas fue superior al $80 \%$ con los dos insecticidas. Se encontró un desplazamiento de los mosquitos del interior al exterior de las viviendas después de la aplicación de los insecticidas. A pesar del impacto inmediato de los insecticidas, no se observó una disminución sostenida de la densidad de adultos a lo largo de las 5 semanas de evaluación post-tratamiento, debido probablemente a la emergencia de adultos de los criaderos, los cuales no fueron tratados.

Conclusión Los insecticidas mostraron una eficacia superior al $80 \%$ en reducción de las densidades de A.aegypti y se constituyen en una alternativa para el control de Dengue.

Palabras Clave: Dengue, insecticidas, control, Aedes, Colombia (fuente: DeCS, BIREME).

\section{ABSTRACT}

Objective Evaluating the efficacy of two pyrethroid insecticides on Aedes aegypti, thereby providing useful information for decision-making in controlling dengue. Methods The effect on $A$. aegypti of ultra low volume (ULV) highcis-Permethrin (Depe) and $ß$-cypermethrin pyrethroids in a smoke-generating formulation (Bolate) 
was evaluated in 16 blocks in the town of Puerto Leguizamo, Putumayo, between July and December 2004. Their efficacy was measured by the mortality of mosquitoes kept in sentinel cages set in different parts of the households and weekly follow-up of the number of mosquitoes and the presence of larvae.

Results There was an $80 \%$ reduction in mosquito density on the day after insecticide treatment for both insecticides. Mosquitoes became displaced from inside the houses to outside them after the insecticides had been applied. In spite of the insecticides' immediate impact, a maintained reduction in mosquito density throughout the 5 weeks' evaluation was not observed, probably due to the emergence of adults from deposits which had not been treated.

Conclusion These insecticides showed higher than $80 \%$ efficacy in reducing $A$. aegypti mosquito density and they constitute an alternative for dengue control.

Key Words: Dengue, insecticide, control, Aedes, Colombia (source: MeSH, NLM).

$\mathrm{E}$ l dengue es la enfermedad más importante transmitida por vectores en las áreas urbanas tropicales (1). En Colombia la incidencia del dengue clásico y dengue hemorrágico se ha caracterizado por la presentación de brotes epidémicos en gran parte de las poblaciones urbanas localizadas por debajo de los 2000 m.s.n.m. Debido a los altos índices de infestación por Aedes aegypti en la mayoría de municipios colombianos, a la circulación de varios serotipos del virus del dengue y el número de personas susceptibles al serotipo 3 que habitan estas zonas, una gran parte de la población del país está en riesgo de desarrollar estas enfermedades (2)

Las estrategias para el control de dengue se dirigen principalmente hacia lograr la participación comunitaria para reducir las fuentes de cría de los mosquitos vectores. Sin embargo, ante epidemias de dengue clásico o hemorrágico, una de las acciones prioritarias a realizar por las autoridades de salud es el uso de insecticidas con el fin de interrumpir rápidamente la transmisión, al disminuir las densidades de adultos de su vector, A. aegypti. Los insecticidas usados en Colombia para el control de epidemias de dengue y dengue hemorrágico son principalmente insecticidas organofosforados como son el malation y temefos contra los adultos y fases larvarias respectivamente.

En este trabajo se evaluaron los piretroides highcis-permetrina (Depe ${ }^{\circledR}$ ) con 3 aplicaciones siguiendo las recomendaciones de la OMS para casos de epidemias (días 1,4 y 7) y ß-cipermetrina, en su presentación de Pote Fumígeno con una única aplicación, con el fin de disponer de información sobre su eficacia para el control de A. aegypti en Colombia. 
Highcis-permetrina contiene permetrina con una relación de isomeros cis superior al $80 \%$. Este piretroide se desarrolló y se ha evaluado en Argentina contra A. aegypti con resultados promisorios (3). El piretroide ß-cipermetrina se incorporó en una presentación novedosa de aplicación de insecticidas para el intradomicilio, un pote fumígeno. Este es un recipiente con una mecha que al encenderla genera rápidamente por combustión una gran cantidad de humo a presión que arrastra los ingredientes activos de ß-cipermetrina y los distribuyen homogéneamente en el ambiente tratado permitiendo una rápida penetración a lugares de difícil acceso. El pote fumígeno ha sido utilizado para el control de triatomineos en Argentina (4) y muestra potencial para ser usado en el control de A. aegypti (5). La utilización de este método en campañas de control de dengue ofrecería como ventaja la participación comunitaria, ya que los habitantes de las casas pueden utilizarlo, previo entrenamiento, sin necesidad de la presencia de funcionarios de salud en cada vivienda, lo que conlleva a una reducción de costos operativos y de equipos para aplicación del insecticida.

\section{METODOLOGÍA}

Área de estudio

La evaluación se realizó en la cabecera municipal de Puerto Leguízamo en el Departamento de Putumayo, entre julio y diciembre del 2004. Este municipio se encuentra a 200 m.s.n.m, con una temperatura media de $27^{\circ} \mathrm{C}$ y aproximadamente 30725 habitantes. Aunque P. Leguizamo es una de las cabeceras municipales que mejores servicios públicos tiene en el departamento del Putumayo, el abastecimiento de agua no es regular, y la comunidad se ve obligada a recolectar agua en diferentes tipos de depósitos, lo cual favorece al incremento de criaderos de los mosquitos $A$. aegypti.

Evaluación pre-tratamiento

Se seleccionaron 16 manzanas, a las cuales se les estimó la presencia y densidad de adultos y presencia de larvas en los depósitos durante 5 semanas pretratamiento. Cada semana se visitó el $25 \%$ de las casas, 8 casas en promedio, de cada manzana. Cada semana se inspeccionaron 1 de cada 4 casas en cada manzana, donde una persona recolectó los mosquitos adultos durante 10 minutos de búsqueda activa, con aspiradores manuales. Se realizaron observaciones sobre la presencia de depósitos con larvas de mosquito, y se tomaron muestras de estas para su posterior identificación. 
Se realizó un ordenamiento de las manzanas según la abundancia de mosquitos adultos y en cada categoría se asignó al azar un tratamiento: highcispermetrina (6 manzanas), ß-cipermetrina en pote fumigeno (4 manzanas) y sin intervención o control (6 manzanas).

\section{Tratamiento}

El piretroide highcis-permetrina en formulación de concentrado emulsificable (DEPE ${ }^{\circledR}$ ), se aplicó en 200 casas, con una maquina Twister Dinafog a ultra bajo volumen (ULV- ultra-low volume), siguiendo el esquema empleado en casos de epidemia de dengue, con aplicaciones en los días 1, 4 y 7. La primera aplicación se realizó a una dosis de $150 \mathrm{mg} / \mathrm{casa}$, pero se obtuvieron porcentajes bajos de mortalidad de los mosquitos en las jaulas centinela, por lo que se incrementó ésta dosis a la recomendada por el fabricante: $1400 \mathrm{mg} / \mathrm{casa}$, dosis que fue utilizada en la segunda y tercera aplicación. Las jornadas de fumigación se realizaron los días 5, 8 y 11 de Noviembre de 2004, por funcionarios de DASALUD Putumayo.

El piretroide ß-cipermetrina en pote fumígeno tuvo una aplicación única el día 13 de Noviembre de 2004, en 126 casas. Se colocó, un pote por cada vivienda en la parte central de las casas. Una vez se prendió la mecha del pote, las puertas traseras y principales, al igual que las ventanas exteriores se mantuvieron cerradas. Tanto habitantes como los funcionarios de salud permanecieron alejados de las viviendas por espacio de una hora para evitar quedar expuestos al humo generado. Se les pidió a los habitantes que antes de regresar a las casas dejaran ventilar las viviendas por un tiempo superior a media hora.

Se utilizaron jaulas centinela de $20 \times 20 \times 3 \mathrm{~cm}$, con de 14 a 17 hembras de A. aegypti, para medir la eficacia del impacto inmediato de los insecticidas. Se tomaron lecturas inmediatas de la mortalidad de los mosquitos expuestos, en cada jaula y a las 24 horas post-exposición.

\section{Evaluación Post-tratamiento}

El día posterior a la aplicación de los insecticidas se realizaron visitas a 5 casas al azar en cada manzana tratada y control, registrándose la cantidad de mosquitos adultos por casa durante diez minutos de búsqueda. El promedio de adultos por día encontrados en manzanas tratadas y control la semana anterior a la aplicación de los insecticidas se comparó con el número de 
adultos encontrados en estas mismas manzanas un día después de la intervención y se calculó el porcentaje de reducción. Este porcentaje fue calculado según la formula de Mulla (6): \% Reducción = 100 - [(C1/C2)x(T2/ T1)] x 100, donde C1: No. de adultos en el control antes de la aplicación, C2: No. de adultos en el control después de la aplicación, T1: No. de adultos en las casas tratadas antes de la aplicación, T2: No. de adultos en las tratadas después de la aplicación.

La estimación de la densidad de adultos y presencia de larvas en los depósitos se realizó durante 5 semanas post-tratamiento. La metodología usada y el esquema de visitas seguido en esta fase del estudio fue el mismo que se siguió para las 5 visitas pre-tratamiento.

\section{RESULTADOS}

Mortalidad en jaulas centinela

Los resultados de las jaulas centinelas expuestas en las manzanas tratadas y control se presentan en la Tabla 1. En la primera aplicación de highcis-permetrina, se observó un porcentaje de mortalidad inferior al control, probablemente debido a la baja dosis aplicada. A partir de la segunda aplicación de highcis-permetrina, en las que se aplicó $1400 \mathrm{mg} / \mathrm{casa}$, se observaron mortalidades superiores al 75 $\%$. La mortalidad en las jaulas colocadas en casas donde se aplicó ß-cipermetrina en pote fumígeno fué de $88 \%$ a las 24 horas posterior a la exposición. Con este tratamiento se observó una mortalidad del $96 \%$ al retirar las jaulas centinela en las horas siguientes a la aplicación.

Densidades de adultos $1^{\text {er }}$ día Post-tratamiento

El número de adultos de A. aegypti encontrados en las casas tratadas y casas control antes y después de la aplicación de highcis-permetrina y ß-cipermetrina en Pote fumígeno se presenta en la Tabla 2. En esta Tabla se muestra la media geométrica, y las comparaciones entre manzanas tratadas y control, además de los porcentajes de reducción para cada tratamiento. Los porcentajes de reducción de adultos de A. aegypti por la aplicación de highcis-permetrina en su segunda y tercera aplicación, así como por la aplicación del pote fumígeno, fueron superiores al $80 \%$. En el día posterior a la primera aplicación de highcispermetrina no se obtuvo reducción al compararla con la semana anterior ($81,7 \%)$, por la dosis tan baja aplicada. 


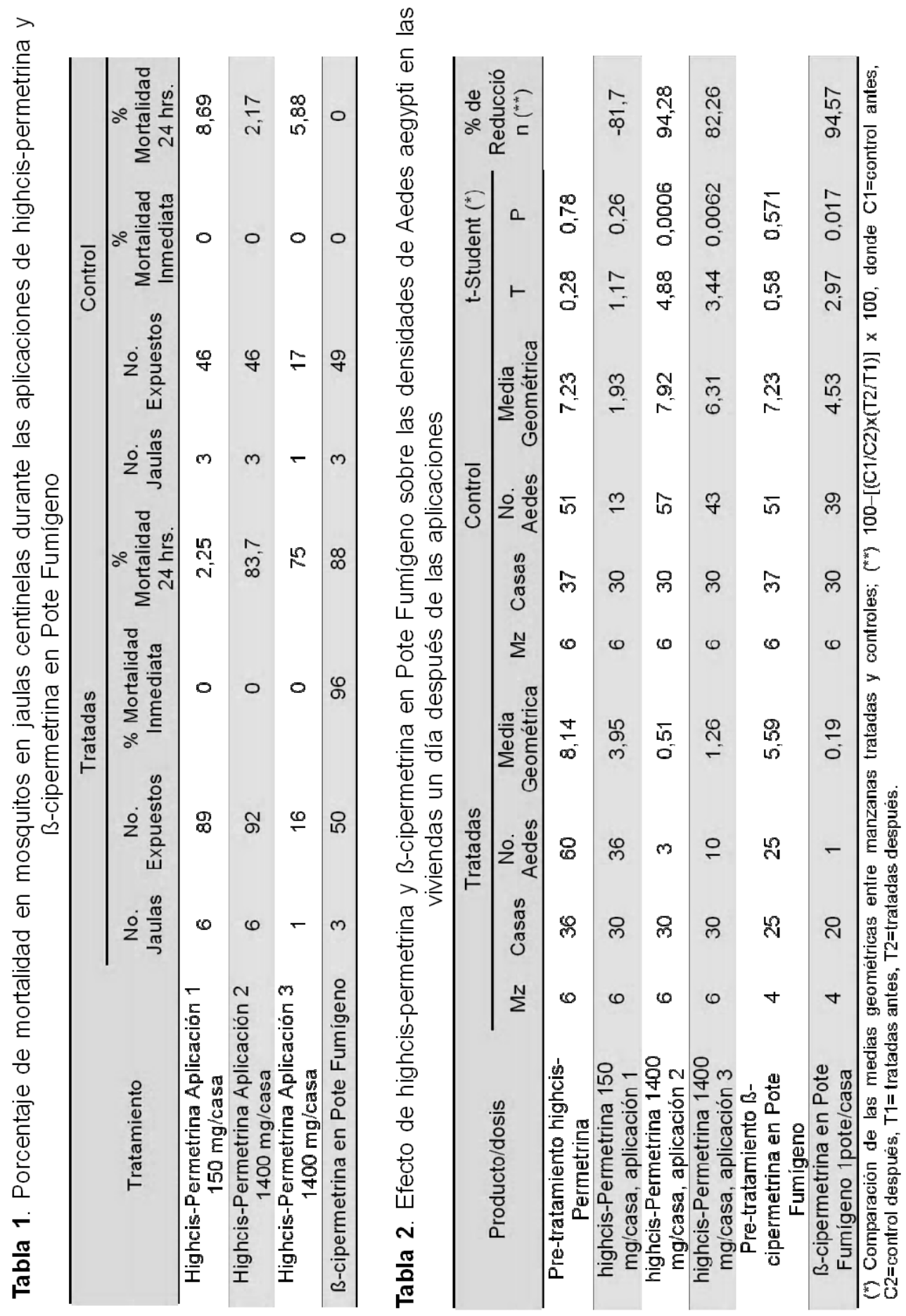


En la Figura 1 se presenta la distribución de A. aegypti en los diferentes cuartos y patio de las viviendas, antes y después de la aplicación de highcispermetrina y ß-cipermetrina en Pote fumígeno. Se observó un cambio en la distribución de los mosquitos al encontrarse adultos en el patio después de la aplicación de los insecticidas, cambio que no se presentó en las casas que no fueron tratadas.

Figura 1. Distribución de las hembras de Aedes aegypti en las viviendas antes y después de los tratamientos con highcis-permetrina (DEPE), ß-cipermetrina en pote fumígeno (POTE) y en las manzanas control

ANTES
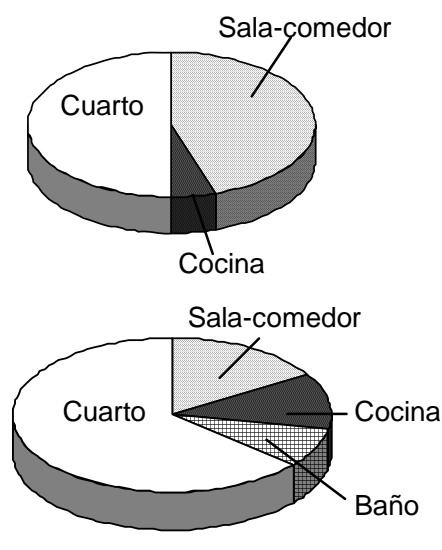

Sala-comedor

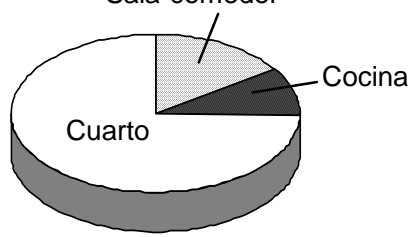

DESPUÉS
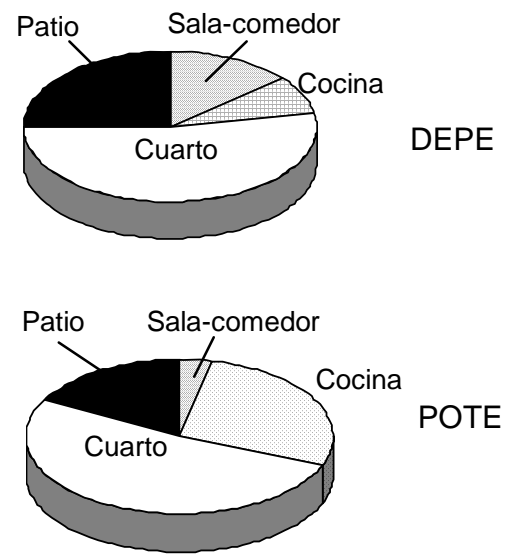

Sala-comedor

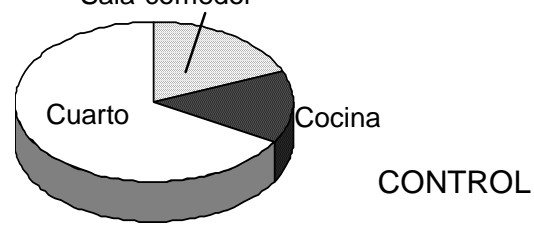

Evaluación de la persistencia del efecto de highcis-permetrina y ßcipermetrina en Pote fumígeno

El seguimiento semanal de las densidades de adultos de A. aegypti antes y después de la intervención con highcis-permetrina y ß-cipermetrina en Pote fumígeno se presentan en la Figura 2. Los resultados no mostraron una disminución significativa de las densidades de adultos de A. aegypti en las 
semanas posteriores a la intervención con los insecticidas. Se compararon los promedios de adultos por casa en las semanas previas y posteriores al tratamiento mediante una T-student (ß-cipermetrina en Pote fumígeno: $t=0.19$; $\mathrm{P}=0.085$ y highcis-permetrina: $\mathrm{t}=0.83 ; \mathrm{P}=0.4316$ ). Tampoco fueron significativas las diferencias en las manzanas control $(\mathrm{t}=-0.15 ; \mathrm{P}=0.8832)$.

Figura 2. Densidades de adultos de Aedes aegypti antes y después de la aplicación de highcis-permetrina y ß-cipermetrina en Pote fumígeno, comparados con las manzanas control

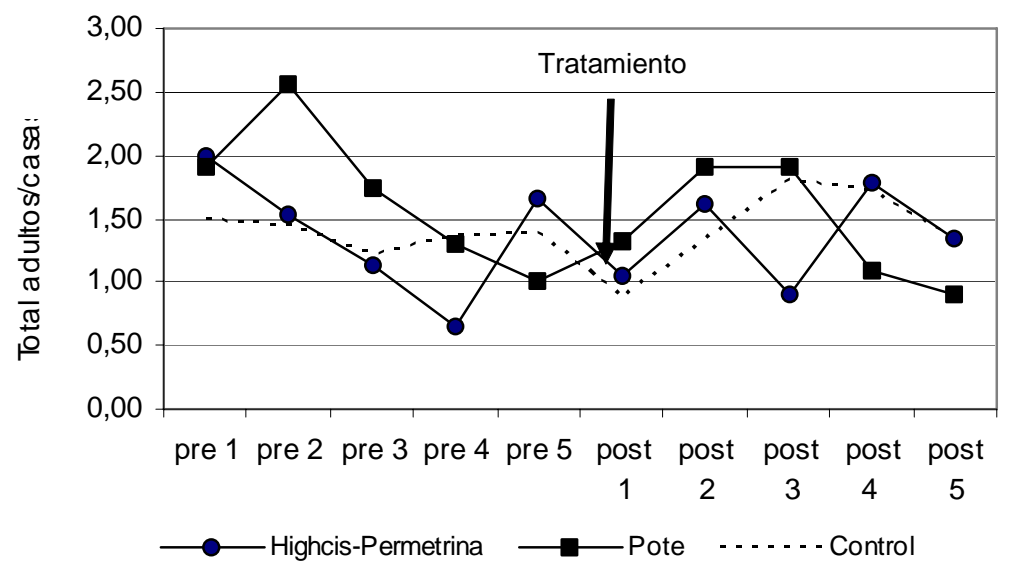

El Indice de Breteau (No. de depósitos positivos/casas evaluadas) mostró una leve tendencia a la disminución posterior a la aplicación de los insecticidas, sin embargo, esta disminución se vio también en las casas control, correspondiendo probablemente a la dinámica natural de la población, mas que a la acción de los insecticidas. No fue evidente una diferencia entre éste Índice en las casas tratadas, respecto a las de control.

\section{DISCUSIÓN}

En este estudio se evaluaron los piretroides highcis-Permetrina (> $80 \%$ cis) y $ß$-cipermetrina en un Pote fumígeno, como una alternativa para el control de A. aegypti. Los dos insecticidas mostraron tener un impacto inmediato sobre las poblaciones de A. aegypti , evidenciado en los resultados obtenidos en las jaulas centinelas expuestas y en el número de adultos encontrados en las casas tratadas al día siguiente de la aplicación. Los porcentajes de reducción de los adultos en las casas, al comparar el promedio del número de adultos/casa antes y el día posterior a la aplicación del insecticida, reportaron valores superiores al $80 \%$. 
Una observación interesante realizada en este estudio fue el desplazamiento de los adultos de A. aegypti desde el interior de las viviendas hacia el patio, observado en las manzanas después de la intervención con highcis-permetrina y con el Pote fumígeno con ß-cipermetrina. Las manzanas control mantuvieron la distribución de los mosquitos adultos en el interior de las viviendas, principalmente en las habitaciones, cocinas etc. encontrándose ausencia de adultos en el patio. Este cambio en los sitios de reposo puede estar significando una acción repelente de estos insecticidas sobre $A$. aegypti, hecho que ha sido documentado en estudios que muestran un impacto de la permetrina no solo a nivel de mortalidad, sino de repelencia y disminuyen tasas de picadura en mosquitos (7), flebotomineos (8) y garrapatas $(9,10)$. También se ha mostrado una mayor acción repelente de Permetrina con respecto al repelente DEET y una mezcla de repelentes (11). Se reporta, similarmente, repelencia de la ßcipermetrina contra Triatoma infestans, incluso mayor que la generada por Deltametrina y Cipermetrina (12). Esta acción repelente es un factor importante para tener en cuenta en los esquemas de aplicación de insecticidas en epidemias de dengue o intervenciones de tipo focal, en donde las aplicaciones se realizan en unas cuantas viviendas alrededor de las casas donde se han presentado casos, dado que los mosquitos adultos podrían desplazarse a los patios o casas cercanas no intervenidas, escapando así del efecto de los insecticidas.

La poca acción de los insecticidas evaluados en este estudio sobre las fases acuáticas del vector era esperada y además considerada por otros autores $(13,14)$, por lo cual se haría interesante evaluar los parámetros estudiados en este trabajo no solo con adulticidas, sino junto con larvicidas de tipo químico y/ o biológico. De esta forma, podría aumentar el efecto de persistencia de los tratamientos al mantener por un periodo de tiempo las poblaciones con densidades bajas.

Los resultados encontrados en este estudio mostraron que los insecticidas piretroides evaluados pueden ser una medida alternativa a los insecticidas organofosforados para reducir las densidades de adultos de A. aegypti. La acción de los adulticidas fue evidente el día de la aplicación y al día siguiente. Sin embargo, no se vio una reducción sostenida de las densidades en las semanas posteriores al tratamiento. Con el fin de obtener un efecto más duradero sobre las densidades de adultos sería necesario evaluar su acción junto con intervenciones en los criaderos, por métodos químicos, físicos y/o biológicos, que permitan un impacto en todas las fases de A. aegypti. El uso del Pote fumígeno se presenta como una alternativa frente al tradicional uso de las 
maquinas ULV, dada su fácil aplicación, su poder de penetración a todos los sitios de la vivienda y la posibilidad de involucrar a la comunidad

Agradecimientos. A DASALUD-Putumayo: Dra. ME Pabón, Sr. JI Palacios y H Erazo, por el apoyo logístico. A J Giraldo, JF Guzmán, L Araujo y LA Rodríguez por la participación en la fumigación. Al Sr.E. Guzman, Alcalde de Puerto Leguizamo, O. Poveda, Secretario de salud, al Sr. M Rincón, Director del hospital Maria Angelines, al Coronel Diago y a los habitantes del Municipio por la colaboración. A la U. de Antioquia y V\&P Management por la financiación.

\section{REFERENCIAS}

1. Kouri G. El dengue, un problema creciente de salud en las Americas. Rev Panam Salud Publica/Pan Am J Public Health. 2006; 19(3):143-145.

2. Boletín Epidemiológico Semanal, SIVIGILA. Semana epidemiológica No. 08. Febrero 22-28 de 2004. [Internet]. Disponible en: www.col.ops-oms.org/sivigila/2004/ bole08_04.htm. Consultado Noviembre 2006.

3. Seccacini E, Masuh H, Licastro S, Zerba E. Laboratory and scaled up evaluation of cis-permethrin applied as a new ultra low volume formulation against Aedes aegypti (Diptera: Culicidae). Acta Tropica. 2006; 97:1-4.

4. Pinto-Dias JC, Zerba E. Emprego de pote fumígeno para proteção de insetário e sua ação residual contra triatomíneos, em condições de laboratorio. Rev Soc Bras Med Trop. 2001; 34(6): 507-510.

5. Masuh H, De Licastro SA, Lopez PA, Vega C, Zerba E. Field evaluation of a smokegenerating formulation containing beta-cypermethrin against the dengue vector in Argentina. J Am Mosq Control Assoc. 2003;19(1):53-7.

6. Mulla MS, Norland RL, Fanara DM, Darwaseh HA and Mckean DW. Control of chironomid midges in recreational lakes. J Econ Entomol. 1971; 64: 300-307.

7. Lindsay IS, McAndless JM. Permethrin-treated jackets versus repellent-treated jackets and hoods for personal protection against blackflies and mosquitoes. Mosq News. 1978; 30(3) 350-356.

8. Dees WH. Preliminary investigations of protective clothing and repellent used against medically important arthropods in the Sinaí desert. New Jersey Mosquito Control Association. Proceedings of $72^{\text {nd }}$ Annual meeting. 1990. p. 239-241.

9. Lane RS. Treatment of clothing with a permethrin spray for personal protection against the western black legged tick, Ixodes pacificus (Acari: Ixodidae). Exp Appl Acarology. 1989; 6: 343-352.

10. Schreck CE Mount GA, Carlson DA. 1982. Wear and wash persistence of permethrin used as a clothing treatment for personal protection against the Lone Star Tick (Acari: Ixodidae). J Med Entom. 1982; 19(2):143-146. 
11. Schreck CE, Haile DG, Kline DL. The effectiveness of permethrin and deet, alone or in combination, for protection against Aedes taeniorhynchus. Am J Trop Med Hyg. 1984; 33(4):725-30.

12. Woods E, Licastro S, Casabé N, Picollo MI, Alzogaray R y Zerba E. Telas impregnadas de ß-cipermetrina: una nueva táctica para el control de Triatoma infestans. Rev. Pan Salud Publica. 1999;6(1):1-7.

13. Reiter P, Nathan M.B. Reiter P, Nathan MB. Guidelines for assessing the efficacy of insecticidal space sprays for control of the dengue vector Aedes aegypti. Geneva: World Health Organization; 2001. (WHO/CDS/CPE/ PVC/2001.1).

14. Chua KB, Chua IL, Chua IE, Chua KH. Effect of chemical fogging on immature Aedes mosquitoes in natural field conditions. Sing Med J. 2005; 46(11):639-44. 